\title{
Excess processing of oxidative damaged bases causes hypersensitivity to oxidative stress and low dose rate irradiation.
}

\section{$\operatorname{AUTHOR(S):~}$}

Yoshikawa, Yukihiro; Yamasaki, Akira; Takatori, Kazuhiro; Suzuki, Masao; Kobayashi, Junya; Takao, Masashi; Zhang-Akiyama, Qui-Mei

\section{CITATION:}

Yoshikawa, Yukihiro ...[et al]. Excess processing of oxidative damaged bases causes hypersensitivity to oxidative stress and low dose rate irradiation.. Free radical research 2015, 49(10): 1239-1248

\section{ISSUE DATE:}

2015-10

URL:

http://hdl.handle.net/2433/202263

\section{RIGHT:}

This is an Accepted Manuscript of an article published by Taylor \& Francis in 'Free Radical Research' on 2015, available online: http://www.tandfonline.com/10.3109/10715762.2015.1061186; ; The full-text file will be made open to the public on $10 \mathrm{Jul} 2016$ in accordance with publisher's 'Terms and Conditions for Self-Archiving'.; This is not the published version. Please cite only the published version.; この論文は出版社版でありません。引用の際には出版社版をご硫認ご 利用ください。 


\section{Excess processing of oxidative damaged bases causes hypersensitivity to oxidative stress and low dose rate irradiation}

Yukihiro Yoshikawa ${ }^{1}$, Akira Yamasaki ${ }^{1}$, Kazuhiro Takatori ${ }^{1}$, Masao Suzuki ${ }^{2}$, Junya Kobayashi $^{3}$, Masashi Takao ${ }^{4}$ and Qui-Mei Zhang-Akiyama ${ }^{1 *}$

1. Laboratory of Stress Response Biology, Graduate School of Science, Kyoto University, Kitashirakawa-oiwakecho, Sakyo-ku, Kyoto 606-8502, Japan 2. Research Program for the Application of Heavy Ions in Medical Sciences, National Institute of Radiological Sciences, Anagawa 4-9-1, Inage-ku, Chiba,263-8555, Japan. 3. Radiation Biology Center, Kyoto University, Yoshida-konoecho, Sakyo-ku, Kyoto 606-8501, Japan. 4. Department of Project Programs, Institute of Development, Aging and Cancer, Tohoku University, Aoba-ku, Sendai 980-8575, Japan

Key words: hOGG1, 8-oxoG, reactive oxygen species, clustered damage, low dose rate radiation, micronuclei

Running title: Susceptible to radiation and oxidative stress by hOGG1 
${ }^{*}$ Corresponding Author:

Qiu-Mei Zhang-Akiyama, Ph.D.

Laboratory of Stress Response Biology, Graduate School of Science, Kyoto University, Kitashirakawa-Oiwakecho, Sakyo-ku, Kyoto 606-8502, Japan

Phone: $+81-75-753-4097 \quad$ Fax: $+81-75-753-4087$

Email: qmzhang@kingyo.zool.kyoto-u.ac.jp

Abbreviations: E.coli, Escherichia coli; $\mathrm{H}_{2} \mathrm{O}_{2}$, hydrogen peroxide; 8-oxoG, 8-oxoguanine; AP-site. apurinic/apyrimidinic-site; DSB, double strand break; BER, base excision repair; ROS, reactive oxygen species 


\section{Abstract}

Ionizing radiation such as X-ray and $\gamma$-ray can directly or indirectly produce clustered or multiple damages in DNA. Previous studies have reported that overexpression of DNA glycosylases in Escherichia coli (E. coli) and human lymphoblast cells caused increased sensitivity to $\gamma$-ray and X-ray irradiation. However, the effects and the mechanisms of other radiation, such as low dose rate radiation, heavy-ion beams or hydrogen peroxide $\left(\mathrm{H}_{2} \mathrm{O}_{2}\right)$ are still poorly understood. In the present study, we constructed a stable HeLaS3 cell line overexpressing hOGG1 protein. We determined the survival of HeLaS3 and HeLaS3/hOGG1 cells exposed to UV, heavy-ion beams, $\gamma$-rays and $\mathrm{H}_{2} \mathrm{O}_{2}$. The results showed that HeLaS3 cells overexpressing hOGG1 were more sensitive to $\gamma$-rays, $\mathrm{OH}^{\cdot}$ and $\mathrm{H}_{2} \mathrm{O}_{2}$, but not to $\mathrm{UV}$ or heavy-ion beams, than control HeLaS3. We further determined the levels of 8-oxoG foci and of chromosomal double-strand breaks by detecting $\gamma-\mathrm{H} 2 \mathrm{AX}$ foci formation in DNA. The results demonstrated that both $\gamma$-rays and $\mathrm{H}_{2} \mathrm{O}_{2}$ induced 8-oxoG foci formation in HeLaS3 cells. hOGG1-overexpressing cells had increased amounts of $\gamma$-H2AX foci and decreased amounts of 8-oxoG foci compared with HeLaS3 control cells. These results suggest that excess hOGG1 removes the oxidatively damaged 8-oxoG in DNA more efficiently and therefore generates more double-strand breaks. Micronucleus formation also supported this conclusion. Low dose rate $\gamma$-ray effects were also investigated. We first found that over-expression of hOGG1 also caused increased sensitivity to low dose rate $\gamma$-ray irradiation. The rate of micronucleus formation supported the notion that low dose rate irradiation increased genome instability. 


\section{Introduction}

Reactive oxygen species (ROS) are generated by exogenous stimuli such as ionizing radiation and various chemical oxidants. ROS are also generated in living cells by normal cellular metabolism [1-3]. ROS generate a wide variety of DNA lesions such as base damages, apurinic and apyrimidinic (AP) sites, and DNA strand breaks [4-8]. These damages may cause loss of genetic stability, and altered cellular regulation associated with aging and many diseases, including cancer [9-12]. Among the damages, 8-oxoguanine (8-oxoG) is an important mutagenic lesion, which can cause cellular mutations and genomic instability [13, 14]. To repair the damages and to maintain genome stability, oxidatively damaged bases are primarily repaired by base excision repair enzymes in most organisms $[10,11,15-17]$. It is well known, that fapy-DNA glycosylase (example: E.coli MutM, human hOGG1) plays an important role in protection against cellular damage by ROS. It removes 8-oxoG, which is paired with cytosine, from damaged DNA $[18,19]$. AP sites arise after removal of 8-oxoG by hOGG1. AP sites inhibit the replication fork and consequently cause cell death and mutations or strand breaks [20].

Ionizing radiation such as X-rays, $\gamma$-rays and $\alpha$-rays, can directly or indirectly produce clustered or multiple damages in DNA, including oxidatively damaged bases [21-23]. Previous studies showed that over expression of E.coli MutM protein in wild-type E. coli cells increased the cell susceptibility to $\gamma$-ray and $\alpha$-ray irradiations [24]. These studies suggested that excessive DNA glycosylases present in living cells would remove damaged bases which were caused by ionizing radiation, and as a result clustered base damages would be converted into DSBs in bacterial cells $[24,25]$. Yang et al. reported that overexpression of human OGG1 in TK6 cells also caused increased sensitivity to $\gamma$-ray irradiation [26]. However, little is known about the effects of recognition and processing in eukaryotic cells of oxidatively 
damaged bases caused by other types of radiation and other ROS such as hydrogen peroxide $\left(\mathrm{H}_{2} \mathrm{O}_{2}\right)$.

Previous studies demonstrated that overexpression of human hOGG1 in E.coli cells could also increase the cell sensitivity under $\mathrm{H}_{2} \mathrm{O}_{2}$ stress conditions [24]. In the present study, HeLaS3 cells which stably overexpressed hOGG1 were established. The susceptibility to $\gamma$-rays and $\mathrm{H}_{2} \mathrm{O}_{2}$ was increased in these hOGG1-overexpressing cells. Moreover, the amounts of 8 -oxoG and $\gamma$-H2AX, and the number of micronuclei, were analyzed. Increases in the amount of $\gamma$-H2AX and the number of micronuclei, and a decrease in the amount of 8-oxoG foci, were detected in the hOGG1-overexpressing cells when exposed to $\gamma$-rays and $\mathrm{H}_{2} \mathrm{O}_{2}$. We found for the first time that hOGG1-overexpressing cells were more sensitive to low dose rate $\gamma$-ray irradiation, and the genome-stability was also decreased.

\section{Materials and methods}

\section{Cell culture}

HeLaS3 cells were grown in Dulbecco's modified Eagle's medium (D-MEM) (Wako) containing L-glutamine supplemented with $10 \%$ fetal bovine serum or FBS at $37^{\circ} \mathrm{C}$ in $5 \%$ $\mathrm{CO}_{2}$ and $100 \%$ humidity [27].

\section{Plasmid}

A plasmid expressing hOGG1-FLAG was constructed as follows: The plasmid pBluescript bearing the human Ogg1-type1a gene was amplified by polymerase chain reaction (PCR) and subcloned into the pTargeT plasmid vector [28].

\section{Transfection and stable cell line construction}

Stable cell lines overexpressing human hOGG1 were produced using the Lipofectamine2000 (Invitrogen) according to the company's guidelines. HeLaS3 90-95\% confluent cells grown in 
24-well plates were transfected with 1-2 $\mu$ g pTargeT-hOGG1-1a plasmid using Lipofectamine 2000 (Invitrogen) according to the manufacturer's recommendations. After transfection, cells were incubated at $37^{\circ} \mathrm{C}$ in a $\mathrm{CO}_{2}$ incubator for $24 \sim 48$ hours. Then the cells were passaged at 1:10 30 into fresh growth medium. The following day, $500 \mu \mathrm{g} / \mathrm{ml}$ Geneticin disulfate was added to the growth medium. The cells were selected in this growth medium containing Geneticin disulfate for 2 weeks at $37^{\circ} \mathrm{C}, 5 \% \mathrm{CO}_{2}$. Several single colonies were then selected and screened by several methods. The clones ("HeLaS3/hOGG1") were maintained in growth medium with $200 \mu \mathrm{g} / \mathrm{ml}$ Geneticin (G418).

\section{Reverse transcriptase-polymerase chain reaction (RT-PCR)}

Total RNAs were isolated from HeLaS3 or HeLaS3/hOGG1-1a cells that were about $90 \%$ confluent with an RNAqueous-4PCR kit (Ambion) according to the manufacturer's instructions. cDNA was prepared by reverse transcription using M-MuLV Reverse Transcriptase (MBI Ferrmentas). Gene transcripts were identified by reverse transcription-polymerase chain reaction. For the Ogg1 gene, 5'-ACACTGGAGTGGTGTACTAGCG-3'/5'-GCCGATGTT GTTGTTGGAGG-3', and for the ape1 gene 5'-ATAGGCGATGAGGATCATGA-3'/5'-CAACATTCTTGGATCGAGCA-3', and for the GAPDH, 5'-CCATGGAGAAGGCTGGGG-3'/5'-CAAAGTTGTCATGGATGACC-3' were used as primers. The amplification was performed with one denaturing cycle at $95^{\circ} \mathrm{C}$ for $5 \mathrm{~min}$, then 35 cycles at $94^{\circ} \mathrm{C}$ for $50 \mathrm{~s}$, at $60^{\circ} \mathrm{C}$ for $50 \mathrm{~s}$, at $72^{\circ} \mathrm{C}$ for $1 \mathrm{~min}$, and one final extension at $72^{\circ} \mathrm{C}$ for $15 \mathrm{~min}$. RT-PCR products were separated by electrophoresis on 1.5\% agarose gels and visualized by ethidium bromide staining. The products were quantitated with Fuji film BAS-2500 image Analyze (Fuji Photo Film). 


\section{Real-Time PCR}

Total RNAs were isolated from HeLaS3 or HeLaS3/hOGG1-1a cells which were about 90\% confluent with an RNAqueous-4PCR kit (Ambion) according to the manufacturer's instructions. cDNA was prepared by reverse transcription using M-MuLV Reverse Transcriptase (MBI Ferrmentas). Real Time PCR was performed using LightCycler96 (Roche) with THUNDERBIRD SYBR qPCR Mix (TOYOBO). The amplification was performed with one denaturing cycle at $95{ }^{\circ} \mathrm{C}$ for $60 \mathrm{~s}$, then 45 cycles at $95{ }^{\circ} \mathrm{C}$ for $15 \mathrm{~s}$, at 60 ${ }^{\circ} \mathrm{C}$ for $30 \mathrm{~s}$, at $72{ }^{\circ} \mathrm{C}$ for $45 \mathrm{~s}$. Gene amplification specificity was verified by melting curve analyses. For the Ogg1 gene, 5'-ACACTGGAGTGGTGTACTAGCG-3'/5'-TAGCTGGAAGTACTTGCGCA-3', and for the GAPDH 5'-CCATGGAGAAGGCTGGGG-3'/5'-CAAAGTTGT CATGGATGACC-3' were used as primers.

\section{Survival assay}

Cells were grown to about $80 \%$ confluence, and then suspended by trypsinization, and the number of cells was counted. The cell suspensions were appropriately diluted and plated in 100-mm dishes. After 16-24 h incubation in $\mathrm{CO}_{2}$ incubator, the cells were irradiated with $\gamma$-ray, UV or heavy-ion beams at the doses indicated in the text. Low dose rate $\gamma$ irradiation $(1 \mathrm{mGy} / \mathrm{min})$ was also carried out. After incubation for 10 days, cells were fixed and stained with crystal violet. For $\mathrm{H}_{2} \mathrm{O}_{2}, \mathrm{H}_{2} \mathrm{O}_{2}$ combined with $\mathrm{Fe}^{2+}$ treatment, the cells were treated with each agent at the indicated concentration for $30 \mathrm{~min}$ and then incubated for 10 days. For UV irradiation, UVC lamp (HITACHI-GL15, $254 \mathrm{~nm}, 1 \mathrm{~J} / \mathrm{m}^{2} \mathrm{~s}$ ) was used for irradiation. To prevent attenuation of UV, the cells were treated without medium. Colonies of $>50$ cells were counted as survivors and the surviving fraction was calculated. 


\section{Irradiation of heavy-ion beams}

The cells were irradiated with heavy-ion beams (290 MeV/nucleon) generated at the Heavy Ion Medical Accelerator in Chiba (HIMAC) at the National Institute of Radiological Science (NIRS) in Japan. The characteristics, irradiation procedures and dosimetry of heavy-ion beams have been described elsewhere [29]. Briefly, the dosimetry was characterized by three different methods: an ionization chamber method, a fluence measurement method using CR39 track detectors and measurement by silicon diode. The linear energy transfer (LET) value and dose rate were estimated to be $13.3 \mathrm{keV} / \mu \mathrm{m}$ and $1.0 \mathrm{~Gy} / \mathrm{min}$. The irradiation doses at the sample position were determined by multiplying the fluence by the LET using the following formula [30] :

$$
\text { Dose }(\mathrm{Gy})=\text { Fluence }\left(\text { ions } / \mathrm{cm}^{2}\right) \times \operatorname{LET}(\mathrm{keV} / \mu \mathrm{m}) \times 1.602 \times 10^{-9}
$$

All irradiation was carried out at room temperature.

\section{Preparation of cell extracts}

Extracts for western blotting: each type of cell was grown to $>90 \%$ confluence in $60-\mathrm{mm}$ dishes. The cells were washed twice with ice-cold phosphate buffered saline (PBS) and collected using a rubber scraper. The cells were suspended in an equal volume of PBS. After adding an equal volume of $2 \mathrm{X}$ sample buffer (4\% SDS, $100 \mathrm{mM}$ Tris- $\mathrm{HCl} \mathrm{pH} 6.8,20 \%$ glycerol, 12\% 2-mercaptoethanol, and BPB), the mixture was sonicated using a Bioruptor (Cosmo Bio) (30 sec ON, $1.5 \mathrm{~min}$ OFF, repeatedly for a total of $15 \mathrm{~min}$ ).

\section{Western blotting analysis}

15-30 $\mu \mathrm{g}$ of each sample was loaded onto a 10\% SDS-polyacrylamide gel and electrophoresed. The proteins were transferred to a nitrocellulose membrane and blocked with Tween-PBS (TBS) (PBS containing 0.1\% Tween 20) and 5\% dry milk. The membrane was 
incubated with a 1:1000 dilution of anti-FLAG M2 antibody (Sigma) overnight at $4{ }^{\circ} \mathrm{C}$. The membrane was washed three times in TBS and incubated with a 1:3000 dilution of HRP-conjugated anti-mouse second antibody overnight at $4{ }^{\circ} \mathrm{C}$. The membrane was washed three times in TBS and analyzed using ECL luminescence reagent (GE Healthcare) and the band was detected using X-ray film (Fujifilm). For UV irradiation, UVC lamp (HITACHI-GL15, $254 \mathrm{~nm}, 1 \mathrm{~J} / \mathrm{m}^{2} \mathrm{~s}$ ) was used for irradiation. To prevent attenuation of UV, the cells were treated without medium. After irradiation, sample extraction and western blotting assay were performed.

\section{Detection of cellular localization of hOGG1-FLAG}

Cells were seeded in $3.5-\mathrm{cm}$ glass bottom dishes. When the cell density reached about $80 \%$ confluency, the cells were washed with PBS, and fixed with $4 \%$ formaldehyde in PBS for 30 min. Then the cells were washed with PBS for $5 \mathrm{~min}$, and treated with $0.2 \%$ Triton $\mathrm{X}-100$ in PBS for 2 min to enhance antibody penetration. The cells were blocked at room temperature for 15 min with $10 \%$ goat serum in $\mathrm{TN}$ buffer $(0.1 \mathrm{M}$ Tris- $\mathrm{HCl} \mathrm{pH} 7.5,0.15 \mathrm{M} \mathrm{NaCl})$, and then incubated with FLAG M2 mouse antibody (Sigma) in TNT buffer (TN buffer containing $0.05 \%$ Tween 20 ) for 1 hour at room temperature. The cells were washed 3 times with PBS and treated with Alexa Fluor 488-conjugated goat anti-mouse IgG antibody (Invitrogen) in TNT buffer at room temperature for 1 hour. Then fluorescence was observed using a fluorescence microscope (LX70, Olympus).

\section{Observation of 8-oxoG foci formation}

Cells were seeded in $3.5-\mathrm{cm}$ glass bottom dishes $\left(1 \times 10^{5}\right.$ cells/dish $)$ and incubated in D-MEM containing $10 \%$ serum for $24-48 \mathrm{~h}$. After the cells were treated with $\mathrm{H}_{2} \mathrm{O}_{2}, \mathrm{UV}, \gamma$-ray or heavy-ion beams, the cells were further cultured in D-MEM containing 10\% serum for 3 
hours. The cells were washed 3 times with $2 \mathrm{ml} \mathrm{PBS}$ and fixed with $2 \mathrm{ml}$ ice-cold methanol for $20 \mathrm{~min}$ on ice. Then the fixed cells were washed twice with $2 \mathrm{ml}$ PBS at room temperature, and treated with $100 \mu 1 \mathrm{RNase}(10 \mu \mathrm{g} / \mathrm{ml})$ at $37^{\circ} \mathrm{C}$ for 1 hour. The cells were permeabilized by treatment 3 times for 3 min each with $2 \mathrm{ml}$ PBD (PBS containing 0.1\% Tween 20, 0.1\% Triton X-100), and then treated for 30 min with $2 \mathrm{ml}$ of $2 \mathrm{M} \mathrm{HCl}$ and finally with $2 \mathrm{ml}$ of 50 $\mathrm{mM}$ Tris for $10 \mathrm{~min}$. The cells were blocked at room temperature with $100 \mu \mathrm{l}$ Block Ace for $30 \mathrm{~min}$, then $100 \mu \mathrm{l}$ of the diluted primary antibody (8-oxoG antibody(JaICA): Block Ace $=1: 100$ ) was added, and the cells were incubated with the antibody for 2 hours at room temperature. Then the cells were washed 3 times with $2 \mathrm{ml}$ PBS, and $100 \mu 1$ Alexa Fluor 488-conjugated goat anti-mouse IgG antibody (Invitrogen) (secondary antibody: Block Ace $=1: 300$ ) was added, and the cells were incubated with this antibody solution at $4^{\circ} \mathrm{C}$ in the dark for 16 hours. The cells were washed 3 times with $2 \mathrm{ml} \mathrm{PBS}$, and then observed under a microscope (LX70, Olympus). The conditions of exposure to various stresses were as follows: $\gamma$ ray: $5 \mathrm{~Gy}$; Low dose rate $\gamma$ ray: $6 \mathrm{~Gy} ; \mathrm{H}_{2} \mathrm{O}_{2}: 300 \mu \mathrm{M}, 30 \mathrm{~min}$; UV. $15 \mathrm{~J}$; heavy-ion beams,

\subsection{Gy [31].}

\section{Double-strand break detection}

The cells were grown in $3.5-\mathrm{cm}$ dishes until they reached a density of $2 \times 10^{5} \mathrm{cells} / \mathrm{dish}$. The cells were irradiated with 3 Gy of $\gamma$-rays (at $0.8 \mathrm{~Gy} / \mathrm{min}$ ). Then irradiated and unirradiated cells were incubated at $37^{\circ} \mathrm{C}$ for the indicated times. The cells were fixed with $4 \%$ formaldehyde in PBS for 20 min. Then they were washed with PBS and treated with $0.2 \%$ Triton X-100 in PBS for 2 min, washed with PBS twice, and incubated with 10\% normal goat serum in TN buffer. The cells were incubated with a 1:500 dilution of anti- $\gamma-\mathrm{H} 2 \mathrm{AX}$ mouse antibody (Upstate Inc.) in TNT buffer for $1 \mathrm{hr}$ at room temperature. The cells were washed three times with PBS and incubated with 1:500 dilution of Alexa Fluor 488-conjugated 
anti-mouse antibody (Molecular Probes) in TNT buffer for $1 \mathrm{hr}$ at room temperature. The cells were washed three times with PBS, stained with $1 \mu \mathrm{g} / \mathrm{ml}$ DAPI for $20 \mathrm{~min}$ at room temperature, and visualized by fluorescence microscopy. At least 200 cells were scored for each experiment.

\section{Measurement of the rate of micronucleus formation}

The cells were cultured until they were about $30 \%$ confluent, and then the culture medium was supplemented with $2.5 \mathrm{mM}$ thymidine (final concentration), and culturing was continued for $24 \mathrm{~h}$. The cells were washed twice with PBS, then fresh culture medium was added to the dishes, and the cells were incubated for $10 \mathrm{~h}$. Then $1 \mathrm{mM}$ hydoroxyurea (final concentration) was added to the culture medium, and incubation was carried out for 14-16 h. After washing the dishes twice with PBS, fresh culture medium was added. The cells were irradiated with $\gamma$-ray at 2 Gy or exposed to $300 \mu \mathrm{M} \mathrm{H}_{2} \mathrm{O}_{2}$, and then cultured for $24 \mathrm{~h}$. The cells were fixed with $4 \%$ formaldehyde in PBS for 10 min, and permeabilized with $0.5 \%$ Triton X-100 in PBS for 5 min. DNA was stained using DAPI, and the rate of micronucleus formation was detected by microscopic observation.

For low dose rate $\gamma$-rays, the cells were irradiated with $\gamma$-rays at $1.5 \mathrm{~Gy}$, and then cultured for $24 \mathrm{~h}$. The cells were fixed with $4 \%$ formaldehyde in PBS for $10 \mathrm{~min}$, and permeabilized with $0.5 \%$ Triton $\mathrm{X}-100$ in PBS for $5 \mathrm{~min}$. DNA was stained using DAPI and the rate of micronucleus formation was detected by microscopic observation. At least 200 cells were scored for each experiment.

\section{Data analysis}

We calculated standard errors and carried out Student's t-test for statistical test. 


\section{Results}

\section{Construction of hOGG1 overexpression HeLaS3 cell lines}

Our previous study showed that E.coli cells overexpressing hOGG1 were more susceptible to ionizing radiation than control E.coli cells [24]. To elucidate the mechanism of the radiation sensitivity and to investigate how the cells respond to $\mathrm{H}_{2} \mathrm{O}_{2}$, heavy-ion beams and low dose rate irradiation, we constructed a plasmid bearing the hOGG1 gene. HeLaS3 cells were cultured and transfected with hOGG1-1a plasmid containing the FLAG tag at the C-terminal using Lipofectamine 2000. Geneticin-resistant colonies were picked up and grown in D-MEM, 10\% serum with 500 1000 $\mu \mathrm{g} / \mathrm{ml}$ Geneticin. After three rounds of extensive screening, we established HeLaS3/hOGG1-1a cells, which stably overexpressed hOGG1-1a. Several clones of HeLaS3 cells overexpressing hOGG1-1a were obtained. The candidate clones were confirmed by several methods.

Overexpression of hOGG1-1a in HeLaS3 cells was checked by RT-PCR, Real Time PCR, western blotting and immunofluorescence staining. Total RNAs were isolated from control HeLaS3 cell and HeLaS3/hOGG1-1a cells. Reverse transcription of the RNA samples was performed using an RNAqueous-4 PCR kit (Ambion Inc.) according to the manufacturer's instructions. Then PCR was carried out using hOGG1 primers and control GAPDH primers. The PCR products were separated by electrophoresis on a $1.5 \%$ agarose gel and quantified by comparison with the GAPDH products. The results showed that the hOGG1 level was increased 2.5 fold in the transfected cell line compared with the control HeLaS3 cells (Figure.1a). Real Time PCR was also carried out using cDNA which used in reverse transcriptional PCR.

The results showed that the hOGG1 level was increased 1.6 fold in the transfected cell line (Figure.1b). 
The transfected HeLaS3 cell line expressed hOGG1-FLAG fusion protein, which could be detected by western blotting using anti-FLAG antibody. The anti-FLAG-stainedband was detected in the extract of the HeLaS3/hOGG1 overexpression cells (Figure.1c).

hOGG1-typela has a strong nuclear localization signal and a weak mitochondrial targeting sequence, and is mainly localized to nuclei $[23,32]$. We therefore examined the subcellular localization of the transfected hOGG1-1a proteins by performing immunofluorescence staining using anti-FLAG antibody. As shown in Figure.1d, FLAG-tagged hOGG1 was localized mainly in the nucleus in HeLaS3/hOGG1 cells. The HeLaS3 cells overexpressing hOGG1-1a were therefore used for the following experiments.

Figure 1

\section{Overexpression of hOGG1 increased the cell susceptibility to $\gamma$-ray irradiation and hydrogen peroxide treatment}

Previous studies showed that overexpression of hOGG1 increased the cell sensitivity to $\gamma$-ray irradiation in E. coli cells and human TK6 cells [24, 26]. First, we investigated whether overexpression of hOGG1 caused radiosensitivity in HeLaS3 cells. $\quad \gamma$-ray survival was measured by a colony-formation assay. The results in Figure. 2a show that hOGG1-overexpressing HeLaS3 had enhanced cell sensitivity to $\gamma$-irradiation. $\gamma$-rays generate reactive oxygen species and produce clustered damage to DNA.

We further investigated the effect of $\mathrm{H}_{2} \mathrm{O}_{2}$ on HeLaS3 cell lines and HeLaS3/hOGG1 cells. The results showed, when the cells were treated with $100 \mu \mathrm{M}, 200 \mu \mathrm{M}$ or $300 \mu \mathrm{M} \mathrm{H}_{2} \mathrm{O}_{2}$, overexpression of hOGG1 increased the cell sensitivity to $\mathrm{H}_{2} \mathrm{O}_{2}$ (Figure. 2b). On the other hand, cells exposed to UV showed similar sensitivity between HeLaS3 and HeLaS3/hOGG1 
cells (Figure.2c). Subsequently, we investigated the effect of $\mathrm{OH}^{*}$ on cell survival after treatment of the cells with $\mathrm{H}_{2} \mathrm{O}_{2}$ and $\mathrm{Fe}^{2+}$. The results showed, overexpression of hOGG1 increased the cell sensitivity to $\mathrm{OH}^{\bullet}$ (Figure. 2d).

The above results suggested that processing of oxidative damage by hOGG1 contributes to sensitivity to oxidative stress.

Figure 2

\section{8-oxoG was removed efficiently in HeLaS3/hOGG1-overexpressing cells after exposure to $\gamma$-rays and $\mathrm{H}_{2} \mathrm{O}_{2}$}

$\gamma$-ray irradiation damages cellular DNA by directly attacking the DNA backbone and bases or by indirect effects such as generating reactive oxygen species (ROS) by ionizing cellular $\mathrm{H}_{2} \mathrm{O}$ and $\mathrm{O}_{2}$ [33]. Ionizing radiation produces clustered damages, which contain two or more lesions induced within one or two helical turns of the DNA [34-37]. Non-DSB clustered damages induced by ionizing radiation are less readily repaired than isolated lesions [38-42]. The above cell survival data showed that the sensitivity of the hOGG1-overexpressing cells was enhanced against $\gamma$-rays and $\mathrm{H}_{2} \mathrm{O}_{2}$, but not against UV. What are the differences between these stresses or exposures? Formation of 8-oxoG foci was detected by immunofluorescent staining in each type of cells, which were treated with various stresses. As shown in Figure.3b and $3 \mathrm{c}$, the formation of 8-oxoG foci was significantly increased in $\gamma$-ray irradiated and $\mathrm{H}_{2} \mathrm{O}_{2}$-treated HeLaS3 cells. 8-oxoG foci were hardly observed in UV-and heavy-ion beams treated cells (Figure. 3d and 3e). On the other hand, in hOGG1-overexpressing cells, when exposed to $\gamma$-rays and $\mathrm{H}_{2} \mathrm{O}_{2}, 8$-oxoG foci formation was clearly lower than that in HeLaS3 cells exposed to these treatments (Figure. $3 g$ and $3 h$ ). These results suggested that $\gamma$-ray 
irradiation and $\mathrm{H}_{2} \mathrm{O}_{2}$ treatment generated clustered 8-oxoG, and the generation of such 8-oxoG foci was efficiently decreased in hOGG1-overexpressing cells.

Figure 3

\section{hOGG1-overexpressing cells showed increased DSB formation after $\gamma$-ray irradiation}

\section{and $\mathrm{H}_{2} \mathrm{O}_{2}$ exposure}

HeLaS3 cells overexpressing hOGG1 were sensitive to $\gamma$-ray irradiation. We therefore measured the level of DSBs to examine the reason for this higher sensitivity. We checked the formation of $\gamma-\mathrm{H} 2 \mathrm{AX}$, the phosphorylated form of $\mathrm{H} 2 \mathrm{AX}$ histone that is increased in response to DSBs (Figure. 4a). The cells were irradiated with 3 Gy $\gamma$ rays, and then incubated for the indicated times, and the $\gamma \mathrm{H} 2 \mathrm{AX}$ foci were observed by microscopy and the percentage of foci-positive cells was determined. The results are shown in Figure.4a. The peak of the percentage of foci-positive cells in HeLaS3 was observed at 30 min after irradiation, whereas that in HeLaS3/hOGG1-1a cells was observed at 90 min. In HeLaS3/hOGG1-1a cells the amount of $\gamma-\mathrm{H} 2 \mathrm{AX}$ foci at 90 min of incubation was higher than that in HeLaS3 cells. These results suggested that far more DSBs were generated post $\gamma$ irradiation in hOGG1-overexpressing cells compared with the control cells. We carried out detection of phosphorylation of $\mathrm{H} 2 \mathrm{AX}$ after UVC irradiation by western blotting assay. As a result, phosphorylation of H2AX after UVC irradiaton was detected in both HeLaS3 and HeLaS3/hOGG1 cells, no difference was detected obviously (data not shown).

To further support this suggestion, the rate of micronucleus formation was also measured. The cultured cells were synchronized with thymidine and then with hydoroxyurea, and the G1 phase cells were irradiated with 2 Gy $\gamma$-rays or exposed to $300 \mu \mathrm{M} \mathrm{H}_{2} \mathrm{O}_{2}$, and cultured for a 
further 24 hours, then fixed with formaldehyde and stained with DAPI. As shown in Figure. 4c, in OGG1-overexpressing cells, the rate of micronucleus formation was significantly increased by 2 fold compared with that in HeLaS3 cells when exposed to 2 Gy $\gamma$-rays. In response to $\mathrm{H}_{2} \mathrm{O}_{2}$ treatment, the rate of micronucleus formation of the hOGG1-overexpressing cells was about 2.3-fold higher compared with that in HeLaS3 cells. Furthermore, compared with non-treated hOGG1-overexpressing cells, $\gamma$-ray-irradiated cells generated about 8 -fold more micronuclei and $\mathrm{H}_{2} \mathrm{O}_{2}$-treated cells generated about 7-fold more micronuclei. These results of $\gamma$-H2AX and micronucleus formation assays indicated that hOGG1 increases DSBs and genome instability through excess removal of 8-oxoG in oxidative stress conditions.

Figure 4

\section{hOGG1-overexpressing cells are more sensitive to low dose rate $\gamma$-irradiation than}

\section{HeLaS3 cells, but not more sensitive to heavy-ion beams}

Previous studies showed that high LET radiation (for example: $\alpha$-rays and heavy-ion beams) mainly affected cells by direct effects, and the effects of heavy-ion beams were less dependent on oxygen partial pressure [43].We investigated the contribution of oxidative damage induced by a heavy-ion beams. The cells were irradiated with heavy-ion beams at 0,2 , 4 or 6 Gy. The results showed that the hOGG1-overexpressing HeLaS3 cell survival was similar to that of the HeLaS3 cell line when treated with particular heavy-ion beams irradiation (Figure.5b). The micronucleus formation assay did not show a significant difference between hOGG1-overexpressing cells and HeLaS3 cells (Figure.5c).

Furthermore we investigated whether overexpression of hOGG1 confers radiosensitivity against low dose rate $\gamma$-irradiation in HeLaS3/hOGG1 cells. The cells were exposed to low 
dose rate $\gamma$-ray $(1 \mathrm{mGy} / \mathrm{min})$ irradiation up to $6 \mathrm{~Gy}$. The cell survival was measured by colony formation. The results in Figure.5a showed that overexpression of hOGG1 enhanced cellular sensitivity to low dose rate $\gamma$-irradiation. The micronucleus formation assay also showed that overexpression of hOGG1 increased genome instability (Figure. 5c).

Figure 5

\section{Discussion}

It is thought that ionizing radiation produces a unique form of DNA damage called non-DSB clustered damage, which contains two or more lesions induced within one or two helical turns of the DNA [32-34]. It has been shown that non-DSB clustered damages induced by ionizing radiation could be recognized and removed by DNA glycosylases, such as E.coli MutM and hOGG1, and then were converted to DSBs. Therefore, clustered damages might be biologically significant. However, the mechanisms of recognition of the clustered damaged DNA in human cultured adherent cells and the effects of ROS in cells overexpressing hOGG1 have not been fully studied. In addition, the effect of heavy-ion beams has no enough reports. The effects of low dose or low dose rate irradiation have also remained unknown. It has also remained unknown whether ROS-induced clustered DNA damages influence the cell survival. Thus, there are still many questions waiting to be answered.

Many studies using synthetic duplex oligonucleotides containing two closely opposed lesions and purified enzymes have demonstrated that processing of bi-stranded clustered damages by BER enzymes may actually convert non-lethal clustered damages into lethal DSBs [44-46]. The formation of DSBs depended on whether or not the DNA glycosylases were able to recognize and cleave at the sites of both lesions [47]. 
hOGG1-overexpressing lymphoblast TK6 cells were susceptible to $\gamma$-ray irradiation[22]. In the present study, we constructed a stable HeLaS3 cell line which overexpressed hOGG1 (Figure. 1), and examined the cell sensitivity to $\gamma$-ray irradiation and $\mathrm{H}_{2} \mathrm{O}_{2}$ treatment. The results showed that hOGG1-overexpressing HeLaS3 cells were more sensitive to $\gamma$-radiation, $\mathrm{H}_{2} \mathrm{O}_{2}$ and $\mathrm{OH}^{\cdot}$ treatment compared with control HeLaS3 cells (Figure.2a, 2b, 2d). We also investigated the cell sensitivity to UVC irradiation, and the results demonstrated that hOGG1-overexpressing cells showed similar sensitivity to UV radiation compared with control cells. (Figure.2c). It is well known that pyrimidine dimers are the major products formed by UV irradiation [48] and hOGG1 does not remove these kinds of damage. The results shown in Figure.2a, Figure.2b and Figure.2d suggested that the HeLaS3/hOGG1-1a cell line established here can recognize oxidatively damaged DNA.

To explore the reason for this phenomenon, examination of 8-oxoG, which is a major substrate of OGG1, was important. 8-oxoG formation in HeLaS3 cells is markedly increased when the cells are exposed to $\gamma$-rays or $\mathrm{H}_{2} \mathrm{O}_{2}$. We treated the cells with $\mathrm{H}_{2} \mathrm{O}_{2}$ at $37^{\circ} \mathrm{C}$. At high temperature, $\mathrm{H}_{2} \mathrm{O}_{2}$ might attack DNA more actively than at low temperature. This may be the reason why we could detect 8-oxoG foci with $\mathrm{H}_{2} \mathrm{O}_{2}$ treatment. On the other hand, in hOGG1-overexpressing cells, the number of 8-oxoG foci was decreased (Figure.3). These results showed that 8-oxoG was efficiently removed in hOGG1-overexpressing HeLaS3 cells. $\gamma \mathrm{H} 2 \mathrm{AX}$ and micronuclei formation are indicators of DSBs, and both markers were increased in hOGG1-overexpressing cells (Figure.4). The 8-oxoG in the clustered damages caused by $\mathrm{H}_{2} \mathrm{O}_{2}$ and $\gamma$-ray radiation was effectively removed, and as a result DSBs were generated and consequently cell death was induced.

In the environment of outer space, prolonged exposure to various heavy ion species is predicted. In addition to the cosmic radiation that comes from outer space, organisms are 
exposed to various kinds of radiation generated from the ground. In order to elucidate the effects of heavy particle beams, experiments were conducted here using the hOGG1-overexpressing cells. hOGG1-overexpressing cells did not show increased susceptibility to heavy-ion beams (Figure.5b), that is, they not show an increase of 8-oxoG foci (Figure.3e, j). Considering the fact that we detected a large amount of DNA strand breaks caused by heavy-ion beams (Figure.5c), the effect of heavy-ion beams on cells seemed to have a larger contribution via direct damage of DNA than via indirect effects (including generation of ROS).

The effect of low dose and low dose rate radiation is highly controversial. The biological effects of low dose rate ionizing radiation are difficult to measure. In the present study, the results showed that cells overexpressing hOGG1 are more sensitive to low dose rate $\gamma$-rays than HeLaS3 cells (Figure.5a). However, we could not detect 8-oxoG foci formation (data not shown). Why were the hOGG1-overexpressing cells more sensitive to low dose rate radiation than HeLaS3 notwithstanding the lack of 8-oxoG foci accumulation? We consider it likely that low dose rate irradiation generates ROS continuously by reacting with $\mathrm{H}_{2} \mathrm{O}$ in cells. The ROS generate only small amounts of 8-oxoG. hOGG1 has a DNA glycosylase activity and hardly shows AP lyase activity [49]. hOGG1 removes 8-oxoG from damaged DNA, and then generates an AP site. hOGG1-overexpressing cells do not show increased APE1 expression (data not shown). It is possible that the activity of DNA glycosylase caused an accumulation of AP sites, which are would block DNA replication and RNA transcription. These hypotheses are currently under investigation in our laboratory.

This study showed that overexpression of OGG1 and excess removal of 8-oxoG led to genome instability and cell death(Figure6). In this study, hOGG-overexpressing cells were susceptible to $\gamma$-rays, $\mathrm{H}_{2} \mathrm{O}_{2}$, and low dose rate $\gamma$-rays. $\gamma$-rays produce clustered damages, 
including 8-oxoG. $\mathrm{H}_{2} \mathrm{O}_{2}$ produces large amounts of 8-oxoG. Low dose rate $\gamma$-rays might produce ROS, which generate only small amounts of 8-oxoG. The phenotypes of hOGG1-overexpressing cells indicate the types of damage produced by each stress. hOGG1-overexpressing cells can thus be useful for analysis of damages which are induced by various stressors.

Figure 6

\section{Acknowledgements}

We thank Dr. Elizabeth Nakajima and Ms. Ako Matsui for critically reading the manuscript and thank Ms. Ako Matsui for assistance with the Heavy-ion beams and Low dose rate radiation experiments. This study was supported by the Research Project with Heavy Ions at NIRS-HIMAC (12J-393). This work was supported in part by Grants-in-Aid for Scientific Research (\#24510071) from the Ministry of Education, Culture, Sports, Science and Technology of Japan. This work was performed at the Joint Usage/Research Center (Radiation Biology Center), Kyoto University.

\section{Conflict of interest statement}

The authors declare that there are no conflicts of interest. The authors alone are responsible for the content and writing of the paper. 
(a)

HeLas3/

HeLaS3 OGG1

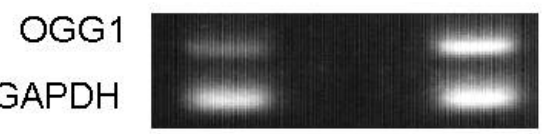

(c)

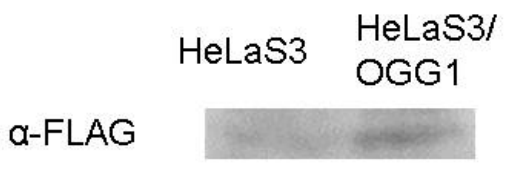

(b)

(d)

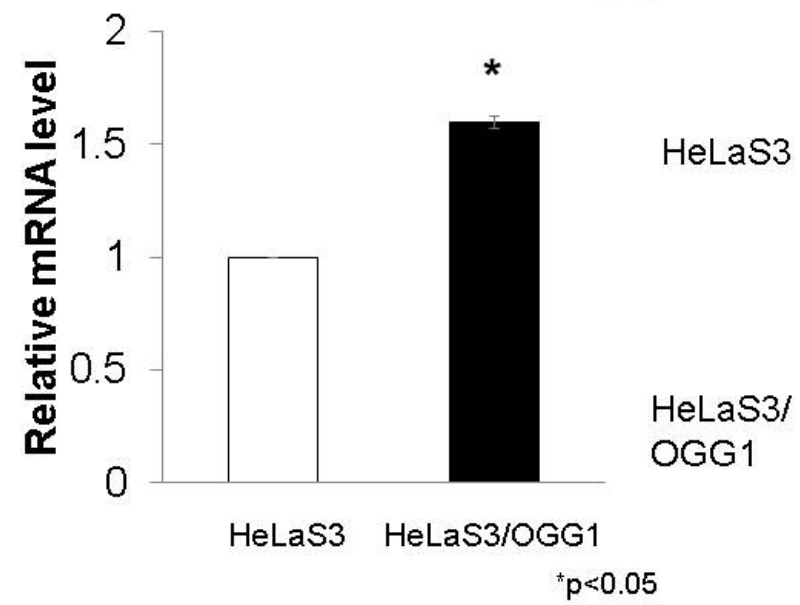

FLAG
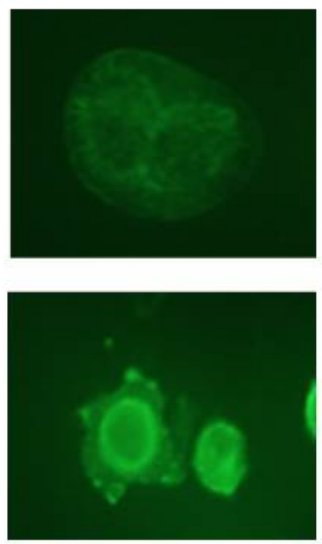

Figure. 1. Overexpression of hOGG1 in HeLaS3 cells (a) Total RNAs were isolated from control HeLaS3 and hOGG1-overexpressing cells. Gene transcripts were detected by RT-PCR. The products were separated by electrophoresis on a $1.5 \%$ agarose gel, and visualized by ethidium bromide staining. (b) Real Time PCR was carried out using cDNA from total RNA. The values represent the mean \pm S.E. $(n=3)$. HeLaS3 cells versus HeLaS3/OGG1 cells by student's t test. (c) hOGG1 protein expression in HeLaS3 was analyzed with anti-FLAG antibody. (d) Localization of overexpressed hOGG1 protein in HeLaS3 cell was confirmed by an immunostaining assay. Immunostaining was performed with anti-FLAG antibody and Alexa Fluor 488-conjugated anti-mouse IgG. The green fluorescence of hOGG1 was observed using microscopy. 
(a)

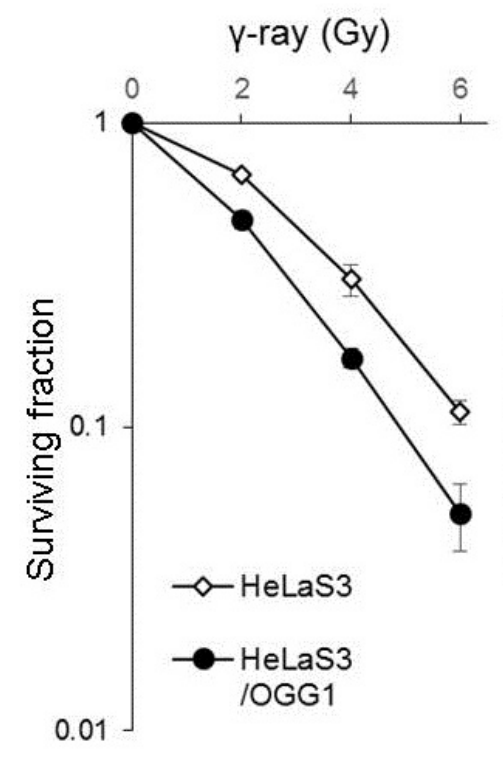

(b)

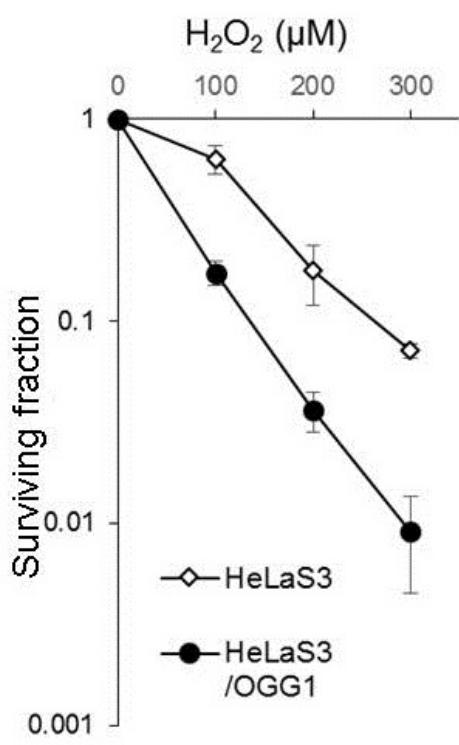

(c)

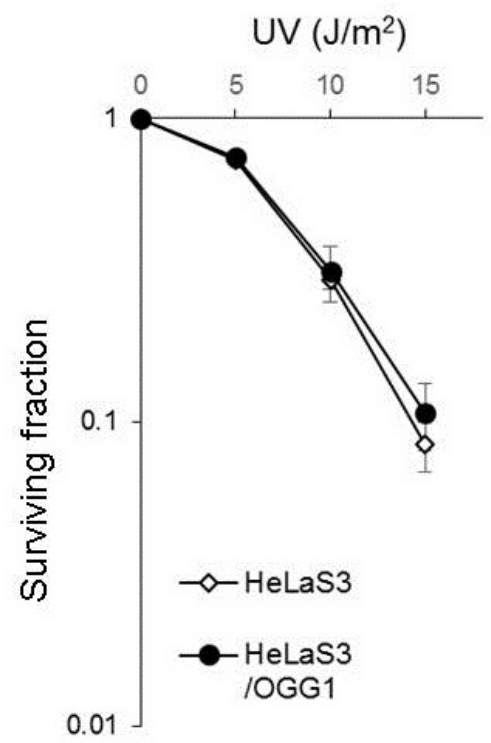

(d)

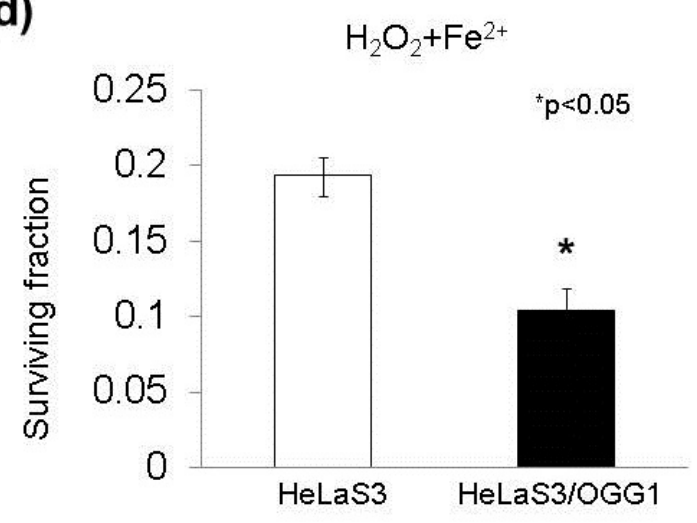

Figure. 2. Survival of HeLaS3 cells and hOGG1-overexpressing cells exposed to various stresses. Exponentially growing cells were appropriately diluted and reseeded in $10-\mathrm{cm}$ culture dishes. Then, the cells were exposed to various stresses. After incubation for 14 days, colonies were counted. (a) Survival of HeLaS3 cells and hOGG1-overexpressing cells following exposure to $\gamma$-rays. (b) Survival of HeLaS3 cells and hOGG1-overexpressing cells following exposure to hydrogen peroxide. (c) Survival of HeLaS3 cells and hOGG1-overexpressing cells following exposure to UV. (d) Survival of HeLaS3 cells and hOGG1-overexpressing cells following exposure to $\mathrm{OH}^{-} \mathrm{H}_{2} \mathrm{O}_{2} 300 \mu \mathrm{M}$ and $\mathrm{FeSO}_{4} 50 \mu \mathrm{M}$. $\mathrm{HeLaS} 3$ cells versus HeLaS3/OGG1 cells by student's $t$ test. The values represent the mean \pm 


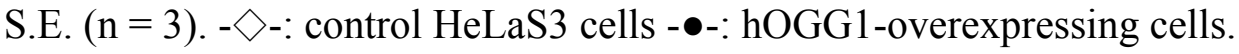

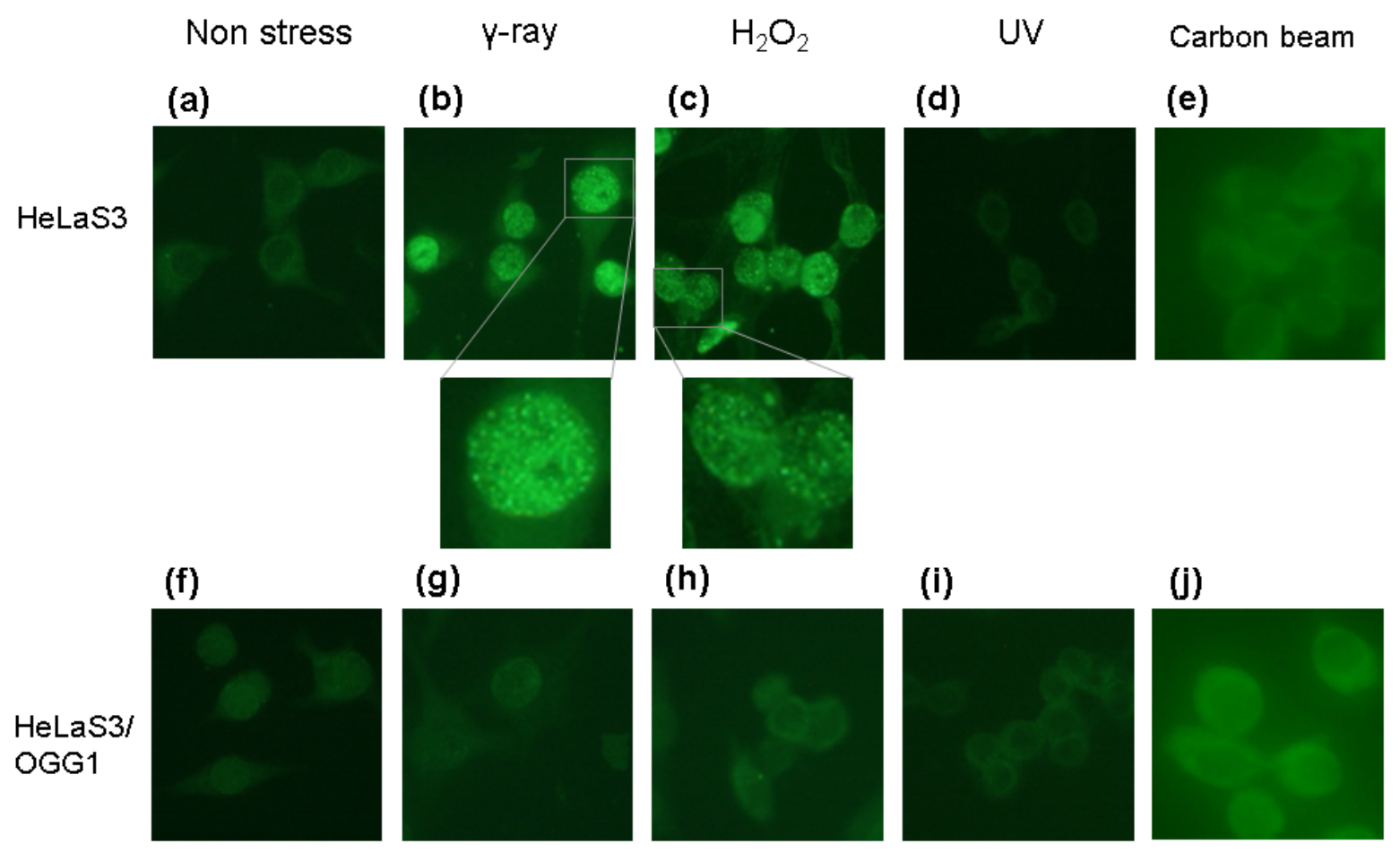

Figure. 3. Immunostaining detection of 8 -oxoG foci following exposure to various stresses. Cells were exposed to 5 Gy $\gamma$-rays (b, g), $300 \mu \mathrm{M}$ hydrogen peroxide(c, h), $15 \mathrm{~J} / \mathrm{m}^{2} \mathrm{UV}$ (d, i), 2 Gy heavy-ion beams $(\mathbf{e}, \mathbf{j})$ or no treatment $(\mathbf{a}, \mathbf{f})$. Immunostaining was performed with anti-8-oxoG antibody and Alexa Fluor 488-conjugated anti-mouse IgG. The green fluorescence corresponding to 8-oxoG was observed using microscopy. 
(a)

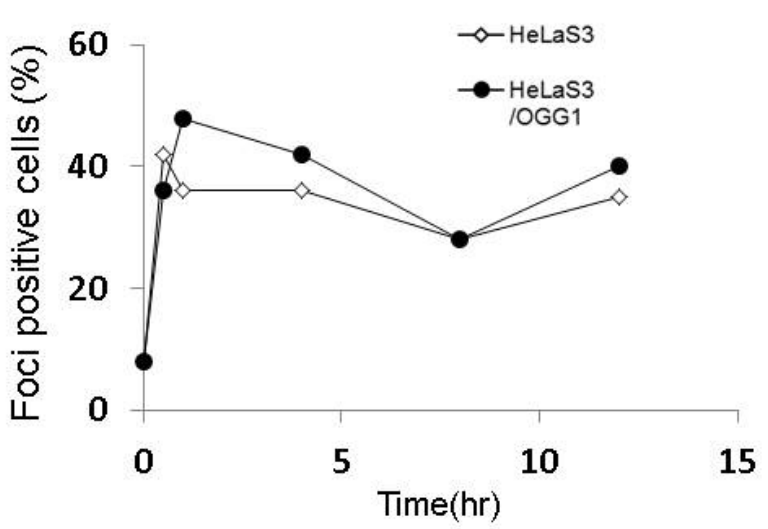

(c)

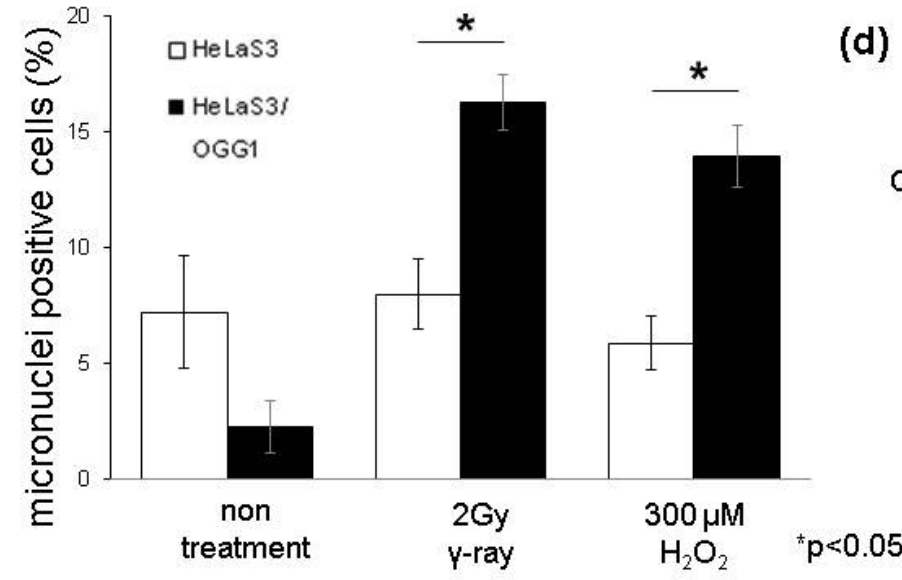

(b)

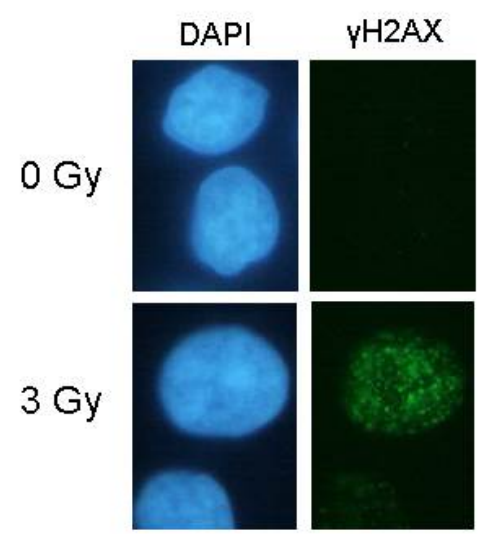

(d)

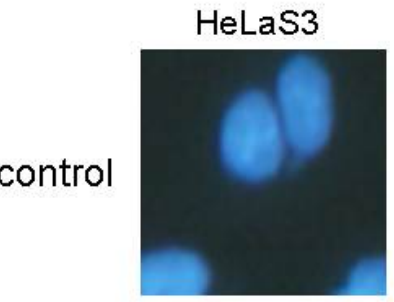

y-ray

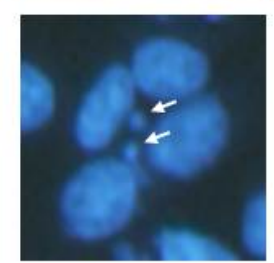

Figure. 4. Analysis of DNA strand break formation and genomic instability by $\gamma \mathrm{H} 2 \mathrm{AX}$ formation and micronuclei formation. (a) Immunostaining detection of $\gamma \mathrm{H} 2 \mathrm{AX}$ foci. Cells were exposed to $\gamma$-rays at $3 \mathrm{~Gy}$. Immunostaining was performed with anti- $\gamma \mathrm{H} 2 \mathrm{AX}$ antibody and Alexa Fluor 488-conjugated anti-mouse IgG. The green fluorescence corresponding to $\gamma \mathrm{H} 2 \mathrm{AX}$ was observed using microscopy. The percentage of $\gamma \mathrm{H} 2 \mathrm{AX}$ foci-positive cells was determined. - $\diamond$-: control HeLaS3 cells - $\bullet$-: hOGG1-overexpressing cells. (b) Immunostaining for $\gamma \mathrm{H} 2 \mathrm{AX}$ in hOGG1-overexpressing cells. (c) DAPI staining detection of micronuclei. Synchronized cells were exposed to $\gamma$-rays at $2 \mathrm{~Gy}$, and hydrogen peroxide at $300 \mu \mathrm{M}$. After further incubation for 24 hours, DAPI staining was performed, and the blue fluorescence of micronuclei was observed using microscopy. HeLaS3 cells versus HeLaS3/OGG1 cells by 
student's t test. (d) DAPI staining to detect micronuclei in HeLaS3 cells.

(a)

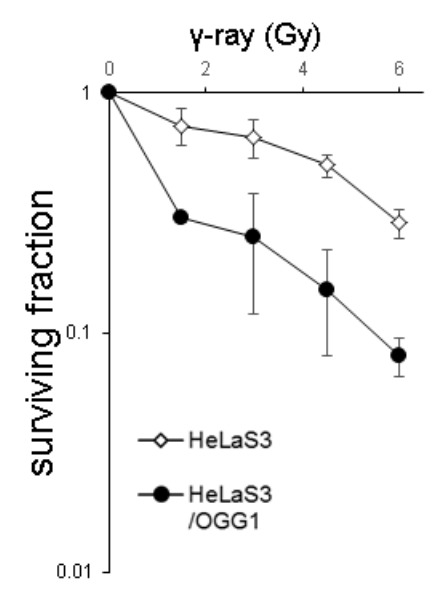

(b)

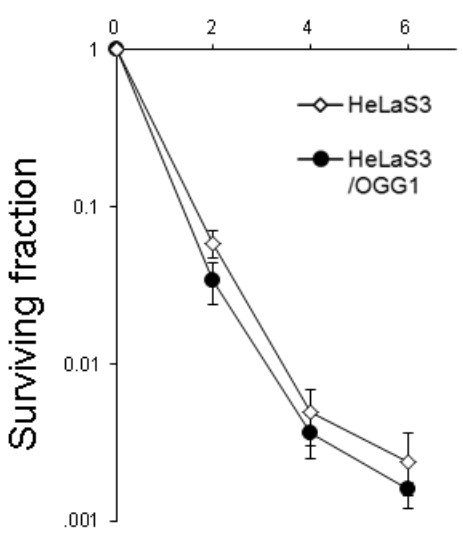

(c)

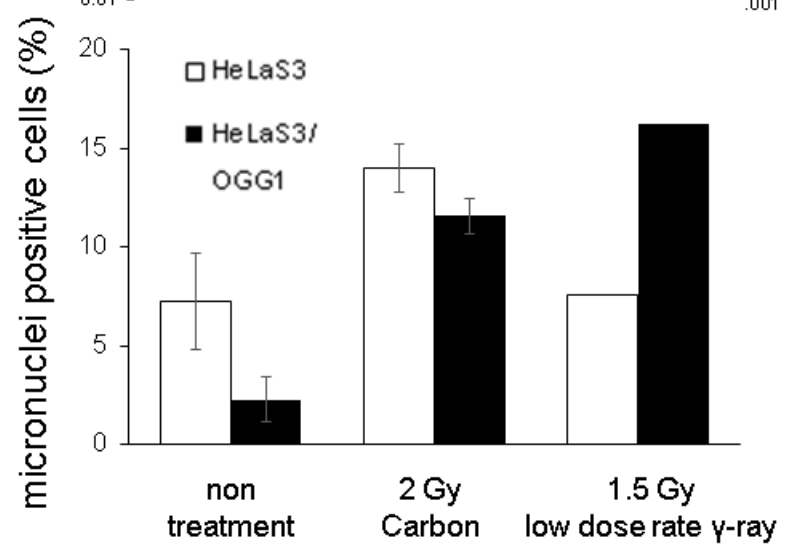

Figure. 5. (a) Survival of HeLaS3 cells and hOGG1-overexpressing cells following exposure to low dose rate $\gamma$-rays ( $1 \mathrm{mGy} / \mathrm{min})$. Exponentially growing cells were appropriately diluted and reseeded in $6-\mathrm{cm}$ culture dishes. After incubation for 10 hours, the cells were exposed to $\gamma$-rays at $37^{\circ} \mathrm{C}$. After exposure to each dose, the cells continued to be incubated in a $37^{\circ} \mathrm{C} \mathrm{CO}_{2}$ incubator. The cells were incubated for a total of 12 days. The values shown represent the mean \pm S.E. $(n=3)$. (b) Survival of HeLaS3 cells and hOGG1-overexpressing cells following exposure to heavy-ion beams. The values represent the mean \pm S.E. $(n=3)$. $-\diamond$-: control HeLaS3 cells - -: hOGG1-overexpressing cells. (c) DAPI staining detection of micronuclei. The cells were exposed to heavy-ion beams at $2 \mathrm{~Gy}$, and low dose rate $\gamma$-rays at $1.5 \mathrm{~Gy}$. After further incubation for 24 hours, DAPI staining was performed, and the blue fluorescence of 
micronuclei was observed using microscopy.

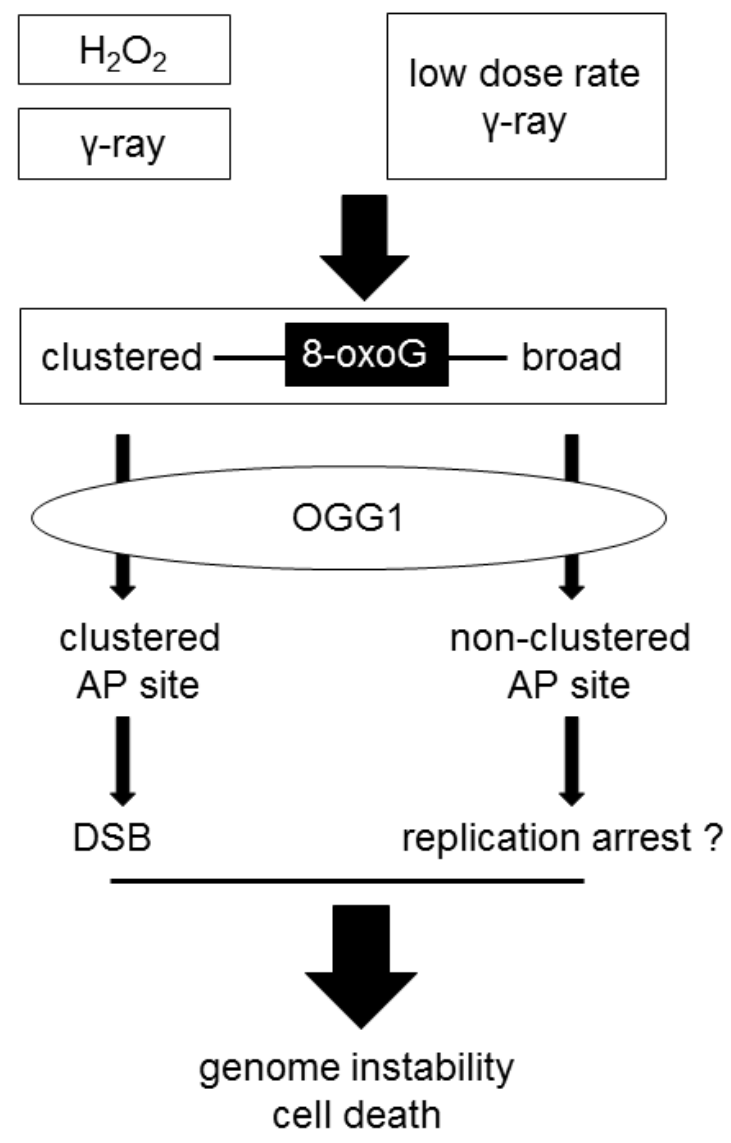

Figure.6. The mechanism of cell death in OGG1-overexpressing cells 


\section{References}

[1] Ames BN, Shigenaga MK, Hagen TM. Oxidants, antioxidants, and the degenerative diseases of aging. Proceedings of the National Academy of Sciences of the United States of America 1993;90:7915-7922.

[2] von Sonntag, C. Topics in Free Radical-mediated DNA Damage: Purines and Damage Amplification-superoxic Reactions-bleomycin, the Incomplete Radiomimetic. International Journal of Radiation Biology 1994;66:485-490.

[3] Choi JY, Cho HJ, Hwang SG, Kim WJ, Kim JI, Um HD and Park JK. Podophyllotoxin acetate enhances $\gamma$-ionizing radiation-induced apoptotic cell death by stimulating the ROS/p38/caspase pathway. Biomed Pharmacother 2015;70:111-8.

[4] Purmal a a, Kow YW, Wallace SS. Major oxidative products of cytosine, 5-hydroxycytosine and 5-hydroxyuracil, exhibit sequence context-dependent mispairing in vitro. Nucleic acids research 1994;22:72-78.

[5] Wallace F. Oxidative Damage to DNA and its repair. 1997. 49-90 p.

[6] David SS, Williams SD. Chemistry of Glycosylases and Endonucleases Involved in Base-Excision Repair. Chemical reviews 1998;98:1221-1262.

[7] Cadet J, Delatour T, Douki T, Gasparutto D, Pouget J P, Ravanat J L, and Sauvaigo S. Hydroxyl radicals and DNA base damage. Mutation research 1999;424:9-21.

[8] Berghian-Grosan C, Radu Biris A, Coros M, Pogacean F and Pruneanu S. Electrochemical and spectroscopic studies of ssDNA damage induced by hydrogen peroxide using graphene based nanomaterials. Talanta 2015;138:209-17. 
[9] Lindahl, T. Instability and decay of the primary structure of DNA. Nature $1993 ; 362: 709-715$.

[10] Beckman KB, Ames BN. The free radical theory of aging matures. Physiological reviews 1998;78(2):547-581.

[11] Marnett LJ. Oxyradicals and DNA damage. Carcinogenesis 2000;21(3):361-370.

[12] Fang W, Deng J, Zhou Y, Lu J, Yang L. A Newfound Association between MDC1

Functional Polymorphism and Lung Cancer Risk in Chinese. PLoS ONE. 2014;9(9):e106794.

[13] Kuipers GK, Slotman BJ, Poldervaart H a., Reitsma-Wijker C a., Lafleur MVM. The influence of combined Fpg- and MutY-deficiency on the spontaneous and $\gamma$-radiation-induced mutation spectrum in the lacZ $\alpha$ gene of M13mp10. Mutation Research DNA Repair 2000;461:189-195.

[14] Coluzzi E, Colamartino M, Cozzi R, Leone S, Meneghini C, O’Callaghan N, Sgura A. Oxidative Stress Induces Persistent Telomeric DNA Damage Responsible for Nuclear Morphology Change in Mammalian Cells. PLoS ONE 2014;9:e110963.

[15] Demple B. and Harrison L. Repair of oxidative damage to DNA: enzymology and biology. Annual Review of Biochemistry 1994;63:915-948.

[16] Mol C.D., Parikh S.S., Putnam C.D., Lo T.P. and Tainer J.A. DNA repair mechanisms for the recognition and removal of damaged bases. Annual Review of Biochemistry 1999;28: 101-128.

[17] Mulder JE, Bondy GS, Mehta R, Massey TE. The impact of chronic Aflatoxin B1 exposure and p53 genotype on base excision repair in mouse lung and liver. Mutation Research 2015;773:63-8.

[18] Michaels ML, Cruz C, Grollman a P, Miller JH. Evidence that MutY and MutM combine 
to prevent mutations by an oxidatively damaged form of guanine in DNA. Proceedings of the National Academy of Sciences of the United States of America 1992;89:7022-7025.

[19] Boiteux S, Radicella JP. The human OGG1 gene: structure, functions, and its implication in the process of carcinogenesis. Archives of biochemistry and biophysics 2000;377(1):1-8.

[20] Boiteux S, Guillet M. Abasic sites in DNA: repair and biological consequences in Saccharomyces cerevisiae. DNA Repair 2004;3:1-12.

[21] O’Neill P. and Fielden E.M. Primary free radical processes in DNA. Advances in Radiation Biology 1993;17:53-120.

[22] Wallace,S.S. Biological consequences of free radical-damaged DNA bases. Free Radical Biology and Medicine 2002;33:1-14.

[23] Shinmura K, Kohno T, Takeuchi-Sasaki M, Maeda M, Segawa T, Kamo T, Sugimura H, Yokota J. Expression of the OGG1-type 1a (nuclear form) protein in cancerous and non-cancerous human cells. International journal of oncology. 2000;16:701-707.

[24] Chang PW, Zhang QM, Takatori K, Tachibana a, Yonei S. Increased sensitivity to sparsely ionizing radiation due to excessive base excision in clustered DNA damage sites in Escherichia coli. International journal of radiation biology. 2005;81:115-123.

[25] Blaisdell, J.O. and Wallace, S.S. Abortive base-excision repair of radiation-induced clustered DNA lesions in Escherichia coli. Proceedings of the National Academy of Sciences of the United States of America 2001;98:7426-7430.

[26] Yang N, Chaudhry MA, Wallace SS. Base excision repair by hNTH1 and hOGG1: A two edged sword in the processing of DNA damage in $\gamma$-irradiated human cells. DNA Repair. 2006;5:43-51.

[27] Hosoki A, Yonekura S-I, Zhao Q-L, Wei Z-L, Takasaki I, Tabuchi Y, Wang L-L, Hasuike S, Nomura T, Tachibana A, et al. Mitochondria-Targeted Superoxide Dismutase 
(SOD2) Regulates Radiation Resistance and Radiation Stress Response in HeLa Cells. Journal of Radiation Research 2012;53:58-71.

[28] Takao M, Aburatani H, Kobayashi K and Yasui A. Mitochondrial targeting of human DNA glycosylases for repair of oxidative DNA damage. Nucleic Acids Research 1998;26:2917-2922.

[29] Kanai T, Endo M, Minohara S, Miyahara N, Kayama-Ito H, Tomura H, Matsufuji N, Futami Y, Fukumura A and Kawachi K. Biophysical characteristics of HIMAC clinical irradiation system for heavy-ion radiation therapy. International Journal of Radiation Oncology Biology Physics 1999;44:201-210.

[30] Suzuki M, Kase Y, Yamaguchi H, Kanai T, Ando K Relative biological effectiveness for cell-killing effect on various human cell lines irradiated with heavy-ion medical accelerator in Chiba (HIMAC) heavy-ion beams. International Journal of Radiation Oncology Biology Physics 2000;48:241-250.

[31] Yoshimura D, Sakumi K, Ohno M, Sakai Y, Furuichi M, Iwai S, Nakabeppu Y. An oxidized purine nucleoside triphosphatase, MTH1, suppresses cell death caused by oxidative stress. Journal of Biological Chemistry. 2003;278(39):37965-37973.

[32] Nishioka K, Ohtsubo T, Oda H, Fujiwara T, Kang D, Sugimachi K, Nakabeppu Y. Expression and differential intracellular localization of two major forms of human 8-oxoguanine DNA glycosylase encoded by alternatively spliced OGG1 mRNAs. Molecular biology of the cell. 1999;10(5):1637-1652.

[33] Halliwell B, Gutteridge J. M.C. Free radicals in biology and medicine $4^{\text {th }}$ edn:Oxford university press;2007;30-78.

[34] Gulston M, Fulford J, Jenner T, de Lara C and O’Neill P. Clustered DNA damage induced by gamma radiation in human fibroblasts (HF19), hamster (V79-4) cells and plasmid 
DNA is revealed as Fpg and Nth sensitive sites. Nucleic Acids Reseach 2002;30:3464-3472. [35] Sutherland B.M, Bennett P.V, Sidorkina O and Laval J. Clustered DNA damages induced in isolated DNA and in human cells by low doses of ionizing radiation. Proceedings of the National Academy of Sciences of the United States of America 2000;97:103-108. [36] Jenner T.J, Fulford J. and O’Neill P. Contribution of base lesions to radiation-induced clustered DNA damage: implication for models of radiation response. Radiation Research, 2001;156:590-593.

[37] Lomax ME, Folkes LK, O’Neill P. Biological consequences of radiation-induced DNA damage: Relevance to radiotherapy. Clinical Oncology. 2013;25:578-585.

[38] Ward J.F. DNA damage produced by ionizing radiation in mammalian cells: identities, mechanisms of formation, and reparability. Progress in Nucleic Acid Research and Molecular Biology 1988;35:95-125.

[39] Goodhead D.T, Thacker J. and Cox R. Effects of radiations of different qualities on cells: molecular mechanisms of damage and repair. International Journal of Radiation Biology 1993;63:543-556.

[40] Ward, J.F. Radiation mutagenesis: the initial DNA lesions responsible. Radiation Research 1995;142:362-368.

[41] Nikjoo H, O’Neill P, Wilson W.E. and Goodhead D.T. Computational approach for determining the spectrum of DNA damage induced by ionizing radiation. Radiation Research $2001 ; 156: 577-583$.

[42] Shikazono N, Noguchi M, Fujii K, Urushibara A, Yokoya A. The yield, processing, and biological consequences of clustered DNA damage induced by ionizing radiation. Journal of radiation research. 2009;50:27-36.

[43] Hirayama R. Mechanism of oxygen effect for photon and heavy-ion beams. Igaku 
Butsuri 2014;34:65-69.

[44] David-Cordonnier M. H, Laval J. and O’Neill P. Clustered DNA damage, influence on damage excision by XRS5 nuclear extracts and Escherichia coli Nth and Fpg proteins. The Journal of Biological Chemistry 2000;275:11865-11873.

[45] David-Cordonnier M.H, Boiteux S. and O’Neill P. Excision of 8-oxoguanine within clustered damage by the yeast OGG1 protein. Nucleic Acids Research 2001;29:1107-1113. [46] David-Cordonnier M.H, Cunniffe S.M, Hickson I.D. and O’Neill P. Efficiency of incision of an AP site within clustered DNA damage by the major human AP endonuclease. Biochemistry 2002;41:634-642.

[47] Harrison L, Hatahet Z. and Wallace, S. S. In vitro repair of synthetic ionizing radiation-induced multiply damaged DNA sites. Journal of Molecular Biology 1999; 290:667-684.

[48] Johns H.E, Pearson M.L, LeBlanc J.C. and Helleiner C.W. The ultraviolet photochemistry of thymidylyl-( $\left.3^{\prime} \rightarrow 5^{\prime}\right)$-thymidine. Journal of Molecular Biology 1964;9:503IN1.

[49] Eccles L.J, O’Nell P, Lomax M.E. Delayed repair of radiation induced clustered DNA damage: friend or foe? Mutation Research 2011;711:134-141. 